\title{
La interculturalidad, perspectivas en el contexto latinoamericano
}

\section{Interculturality, perspectives in the Latin American context}

\author{
Edilberto Valencia Salazar ${ }^{\natural}$ (D) Jacqueline Méndez Gamboa ${ }^{1}$ (ID Segundo E. Vergara Medrano²
}

${ }^{1}$ Universidad Nacional Intercultural de la Amazonia. Pucallpa, Perú

${ }^{2}$ Universidad Nacional de Jaén. Cajamarca, Perú

Recibido: 21/06/2021 Revisado: 02/08/2021_Aceptado: 21/09/2021 Publicado: 31/10/2021

\section{RESUMEN}

El propósito de este artículo es reflexionar sobre interculturalidad como tema emergente en la modernidad, posmodernidad y su alcance, en el actual sistema político, en el contexto Latinoamericano. Del análisis hecho e ideas expuestas se demuestra que, si las políticas públicas interculturales siguen desarrollándose en el actual sistema capitalista, no se podrá lograr los objetivos planificados debido a que, el sistema neoliberal, va generando formas jurídicas capaces de tergiversar el verdadero valor de los elementos culturales de los pueblos originarios (mercancías turísticas o patrimonio cultural de la humanidad). La interculturalidad debe ser una de las políticas públicas prioritarias en la esfera de la educación para el siglo XXI y, debe fundamentarse en; aprender a hacer, aprender a conocer, aprender a vivir juntos y aprender a ser, con más importancia en aprender hacer, es decir, todo individuo debe saber hacer, lo que aprendió y desarrolló en una universidad. Si esto no sucede entonces el sistema universitario habrá fracasado, sobre todo, quienes lo instruyen. Creemos que la interculturalidad funcional está contaminada porque fue desarrollada por y para el estado actual neoliberal. Más bien debe considerarse una interculturalidad objetiva sin ideologías que permita descolonizar el poder del ser y del saber. Una de las formas de ir fertilizando y preparando el terreno para el establecimiento de un nuevo sistema político y social, es generando partidos políticos y movimiento sociales, capaces de incorporar a la interculturalidad como elemento estructural y desarrollar liderazgo inclusivo. Palabras clave: Diversidad, modernidad, interculturalidad, políticas públicas.

\section{ABSTRACT}

The purpose of this article is to reflect on interculturality as an emerging issue in modernity, postmodernity and its scope, in the current political system, in the Latin American context. From the analysis made and ideas presented, it is shown that, if intercultural public policies continue to be developed in the current capitalist system, it will not be possible to develop the planned objectives because, the neoliberal system, is generating legal forms capable of distorting the true value of the cultural elements of the original peoples (tourist goods or cultural he- 
ritage of humanity). Interculturality must be one of the public policies with the highest priority emphasis in the field of education for the 21st century and must be based on; learn to do, learn to know, learn to live together and learn to be, with more importance in learning to do, that is, every individual must know how to do, what he learned and developed in a university. If this does not happen then the university system will have failed and, above all, those who instruct it. We believe that functional interculturality is contaminated because it was developed by and for the current neoliberal state. Rather, it should be considered an objective interculturality without ideologies that allows decolonizing the power of being and knowing knowledge. One of the ways of going under, fertilizing and preparing the ground for the establishment of a new political and social system, is by generating political parties and social movements, capable of incorporating interculturality as a structural element to assume power. And develop inclusive leadership. Keywords: Diversity, modernity, interculturality, public politics.

\section{INTRODUCCIÓN}

En el presente trabajo se analiza desde una perspectiva reflexiva, el desarrollo del enfoque intercultural en el contexto de la modernidad y diversidad en un mundo monocultural y globalizado. Así mismo, se analiza las perspectivas latinoamericanas de la interculturalidad en el futuro por lo menos en el siglo XXI. Así, se pretende responder a la interrogante ¿Cuál es el avance de la interculturalidad en los estados latinoamericanos?, puesto que, en algunos de ellos, se está reconociendo la diversidad cultural en el nivel supremo constitucional, y también conociendo cómo, estas iniciativas, se está plasmando en las políticas públicas. Sabemos que las políticas públicas son formuladas desde los Gobiernos de turno con el propósito de solucionar problemas que se generan en la ciudadanía. Desde esta perspectiva se trata de verificar lo avanzado en Latinoamérica, en general y en el Perú en particular y cuáles son las políticas públicas priorizadas. Por otro lado, frente a las desigualdades sociales y constantes luchas generadas ante este sistema capitalista neoliberal, se están proponiendo movimientos del tipo social y político por los pueblos originarios de tal modo que permita ganar espacios en la participación política en algunos estados.

\section{La modernidad y la diversidad cultural}

Sobre la modernidad en la literatura actual se tiene dos versiones, la primera se gesta en Occidente aproximadamente en el siglo $\mathrm{XV}$, iniciándose con la crisis de la edad medieval, que en su proceso emerge el subjetivismo cartesiano y la objetividad de las ciencias Física-Matemática y, habría llegado a su fin con el ciclo de las grandes guerras europeas de 1914 y 1945 (Daros, 2015). Por su puesto, hay que mencionar los hechos más resaltantes que acompañaron el desarrollo de la modernidad, por ejemplo, el Renacimiento Italiano, Reforma e Ilustración Alemanas y las Revoluciones Burguesas de Inglaterra 1688, de Estados Unidos 1776 y de Francia 1879 y se consolida a partir del siglo XVIII (Aguiló, 2011). La segunda versión es formulada por Enrique Dussel quien argumenta que la modernidad se gesta con la conquista hispano-portuguesa, empezada en 1492 en América, la cual posiciona a Europa como "centro" de la historia (por primera vez) mundial. Esta versión de la modernidad, demuestra su carácter opre- 
sivo y excluyente del indígena. La colonización de América Latina por parte de España y Portugal es una muestra de la expansión del Capitalismo (Mendoza, 2012). Para Marx (Citado por Daza, 2010, p. 72). En la modernidad se evidencia el modo de producción capitalista y las luchas de clases sociales.

En la modernidad se desarrollan relaciones de dominio por parte de la sociedad capitalista quien tiene el poder, un poder en el que están amalgamadas la idea de raza y división del trabajo. La modernidad ha abolido el sistema jurídico y social feudal para proyectar un sistema, individual y capitalista constituyéndose en un proyecto de alcance global. Con la revolución de 1879 surgen los valores que se oponen a la obtención del poder por la fuerza en lo social y político, y así nace el Liberalismo, es decir, la intervención mínima del estado en lo social y económico. Con el constitucionalismo formulado por el liberalismo es como si el mundo estuviera integrado por solo una cultura universal y ha servido para establecer un sistema social, político y económico, pero sin la abolición de clases sociales. Los estados en este sistema son monoculturales y homogéneos, que favorecen a una cultura nacional y no toman en cuenta la diversidad cultural (Aníbal, 214).

En la modernidad también se observa el fenómeno globalizante hegemónico que incorpora a toda la humanidad en un todo. Como lo demuestra la expansión del capitalismo neoliberal en el cual se articula nuevas formas de invasión, expropiación y colonización de territorios de los pueblos originarios. Además, en este muevo contexto los medios de comunicación informáticos reducen el ta- maño del mundo hablando figurativamente, sin embargo, la ventaja es que nos damos cuenta que existen diversas culturas y

formas de vida, lo cual trae como colación el renacimiento de identidades locales, cuya cultura tiene un universo de saberes y conocimientos que tienen que ponerse a prueba. Por tanto, en este nuevo contexto, los conocimientos generados por la ciencia no serían los únicos, sino que habría otros conocimientos, el de los pueblos originarios que habría de someterse a una construcción operacional para validarse. Así mismo ante la diversidad de culturas, la modernidad propone la unidad de la humanidad que cuenta con diferencias culturales en una mono cultura, pero bajo el yugo del mismo sistema político social (Gonzales, 2007).

Lo diverso no debe confundirse con lo diferente. Los seres humanos somos diferentes en lo biológico, social y cultural, así como por el contexto donde vivimos y por la forma como aprendemos. Somos y existimos por la diversidad bilógica. Gracias a la diversidad cultural existen las sociedades. Las desigualdades son engendradas por el sistema político, económico, por prácticas culturales y algunas se crean en las escuelas (Arroyo, 2013). Creemos que es posible suprimir ciertas desigualdades existentes en el sistema global actual, si se elimina al capitalismo neoliberal y se implanta un sistema político, social y económico que busque la construcción de una sociedad con equidad, visibilizando las diferentes culturas. Aquí no se menciona a la democracia como sistema político a tenerse en cuenta, ya que este es un sistema caduco, por ser un sistema donde gobiernan las minorías, basadas en ideologías, como lo es, la democracia 
de la posmodernidad, que niega el derecho a la libertad y el derecho a una educación de calidad, con el sometimiento del individuo a ideologías y subjetividades que lo hacen un ser inútil y servil a los intereses de la elite del poder del capitalismo (Bueno, 2004).

Así mismo, en este marco todas las instituciones de enseñanza-aprendizaje presentan un aspecto plural, por un lado, los estudiantes provenientes de diversas culturas y, por otro, las diferentes formas (estilos) de aprendizaje que manifiestan los estudiantes al momento de procesar y asimilar la información. Debe buscarse un nuevo enfoque en la educación, que tenga en cuenta las dos diferencias (Conde, 2017). En la globalización como parte de esa modernidad, se observa que la diversidad cultural es una cualidad de algunas sociedades en las que generan diversas formas de organización social y política, que ha permitido la convivencia de varias culturas. Sin embargo, la definición de nación ha sido causante de que muchos estados modernos manifiesten una posición hostil con la diversidad cultural (Lanegra, 2016). Para la sociología, esta diversidad global resalta también los problemas sociales, que se conoce por los medios informativos, por ejemplo: las experiencias separatistas de la Yugoslavia, Chechenia, los kurdos de los países árabes, los conflictos de Ruanda, etc. las guerras del medio oriente, aunque resaltan lo económico, pero no hay que ignorar lo cultural y religioso (Gonzales y Sánchez, 2001, citados por Gonzales, 2007). Además, los problemas en las grandes urbes, donde se construyen viviendas en espacios cerrados, con la finalidad de separarse de los vecinos en función a estratos sociales o las diferencias culturales, el problema de los emigrantes en Europa y Estados Uni- dos, y los estigmas de diversos grupos socio culturales, son expresiones violentas que la diversidad global está experimentando (Reguillo, 2005 citado por Gonzales, 2007).

\section{La Interculturalidad en las actuales Políticas Públicas}

La interculturalidad tiene sus orígenes paralelamente tanto en Europa y Latinoamérica en la década de los 80 , primero en el marco educativo y luego se extiende a otras áreas, pero con connotaciones diferentes. Tanto externa como internamente el termino de interculturalidad fue vista primero como multiculturalismo y ha pasado por dos etapas, la primera se usó para apaciguar problemas de conflicto social interno; $y$, en su segunda etapa, se reafirma el término de interculturalidad. En Latinoamérica, la interculturalidad formulada por una de las vertientes más radicales busca la construcción de una convivencia cultural sobre la base de la igualdad social (Mendoza, 2012). Aquí, debe estar implícito el reconocimiento tanto cultural, social como político de los pueblos originarios, a ello, debe agregarse los inmigrantes internos y externos. Habrá que repensar en un concepto que permita polemizar y retar a la dominación en todos sus campos, enfatizando el poder del conocimiento; haciendo notar, al mismo tiempo, el cuestionamiento de la diferencia de poder apoyada por la interculturalidad funcional construida por y para el estado actual, es decir, para un estado neoliberal. Para ello, habrá que revolucionar las estructuras sociales, políticas, gnoseológicas y epistémicas de dominación del saber y del ser, que aún mantiene el modelo capitalista como es el "racismo", considerando a unos seres humanos como inferiores, respecto a las elites (Pérez, 2016), (Millán y Rincón, 2015). 


\section{Sobre definición de interculturalidad}

La interculturalidad es el encuentro entre dos o más culturas, por supuesto suponiendo que hay sociedades multiculturales. Atendiendo a la definición de palabra interculturalidad, la cual proviene del prefijo Inter ("entre" o "medio de"), el núcleo cultura (cultura), aquí parece surgir el problema en la definición de interculturalidad con la de definición de cultura, es confuso ya que el termino cultura tiene diferentes connotaciones, desde el punto de vista social, educativo, económico, político, etc. y que aún falta definir. Así, podríamos atrevernos a decir, que cultura es un concepto local y cambiante, y el sufijo dad que significa cualidad. Así la interculturalidad formaliza la posición y el espacio de reunión y comunicación entre culturas (Hernández, 2007).

Se podría entonces, definir interculturalidad como un proceso dinámico de visibilizacíon de las diferentes culturas para fortalecer el intercambio de conocimientos, saberes y prácticas, con el objeto de contribuir a su desarrollo sostenible tendiente a la construcción de una sociedad con equidad; entonces, debe contextualizarse el problema social y teórico, es decir, se debe tomar o adecuarse a lo que ya existe en otros sistemas sociales-políticos y que han dado resultados, por su puesto contextualizando el tiempo y espacio (Berrospi, 2015). De acuerdo a lo anterior, la interculturalidad puede ser pensada como proyecto social, político y epistemológico, que sucede por la lucha de poder en que se enfrentan procesos de diferenciación, resistencia y sometimiento de los pueblos originarios (Laura, 2004). Aquí, hacemos la observación, de que hay que tener mucho cuidado de exacerbar a la interculturalidad con definiciones que no sean compatibles con la realidad; porque caeríamos en idealismos que a nada bueno nos han llevado y nos llevarían, por ejemplo, el idealismo alemán, causante de las dos guerras mundiales 1914 - 1918 y 1939 -1945.

El sistema político actual, a nivel mundial, es el capitalismo neoliberal que rige en todos sus niveles, en Estados Unidos y sus aliados la Unión Europea, en otros países como China, Rusia, etc. tienen economías mixtas opuestas al neoliberalismo, también encontramos el neoliberalismo en algunos países de Latinoamérica: Colombia, Brasil, Chile, Perú, Paraguay, etc. a excepción de Venezuela y Bolivia (Aguirre, et al. 2020), (Vidal, 2015).

A pesar de los esfuerzos, los esquemas siguen siendo los impuestos por la cultura occidental, el colonialismo, con la diferencia que han creado marcos jurídicos para que pueda apreciarse los elementos típicos y folclóricos de una cultura como mercancía turística inclusive algunos vestigios de la conquista y colonia son declarados patrimonio cultural de la humanidad (Molina, 2012). En ese mismo contexto, las organizaciones representantes de este sistema capitalista, como es la UNESCO, a través de la Comisión Económica para América Latina y el Caribe (CEPAL), reconoce la crisis que está viviendo el sistema neoliberal y proponen una serie de medidas para cerrar brechas frente a la desigualdad y pobreza, para lograr la igualdad (con enfoque de derechos) y sostenibilidad ambiental. Para ello se proponen: cerrar brechas con tasas elevadas; mejor gobernanza de los recursos naturales; mejor repartición de los beneficios entre el capital y la mano de obra (Naciones Unidas, 2014, pp.1-17). Como se observa en la CEPAL se proponen una serie de medidas, pero dentro de su sistema político neoliberal. Lo único que pue- 
den favorecer estas medidas es para que este sistema se siga manteniendo; pero no para generar un cambio, considerando a la diversidad cultural, la igualdad social, etc., como si lo proponen otros sistemas sociales políticos, incluyendo a la interculturalidad, como termino emergente que reclama la reivindicación de los pueblos originarios.

\section{El contexto actual de políticas públicas interculturales}

Antes de describir a las políticas públicas en el contexto intercultural, es pertinente conocer su definición. Según Turner y Hulne (1997) (citado por el Ministerio de Cultura, 2014), considera a una política como un conjunto de objetivos, decisiones y acciones que lleva a cabo un gobierno, junto con el pueblo, para solucionar determinados problemas que, en un determinado momento y espacio, consideran necesario. En realidad, es un proceso complejo en cual se identifican cuatro fases: la agenda, la planificación de estrategias, la implementación de las estrategias y la evaluación de los resultados. Las políticas públicas interculturales deben conducir procesos transformativos a largo plazo. Deben ser aplicados en todas las esferas del estado con una nueva visión de la ciudadanía. Una política intercultural debe liberarse de las singularidades políticas, sociales y económicas que lleven a las desigualdades. Deben ir más allá de búsqueda de la tolerancia y la coexistencia, para alcanzar el respeto y convivencia entre culturas (Cruz, 2013). Para el caso del Perú, el Ministerio de Cultura es el responsable de todo lo concerniente con los aspectos culturales del País, de acuerdo a Ley de Creación N. ${ }^{\circ}$ 29565. Por lo tanto, es correcto asumir que es el "ente rector" en cuanto a cultura e interculturalidad. Dentro del ministerio de cultura, el Viceministro de
Interculturalidad tiene la función de formular políticas, programas y proyectos que promuevan la interculturalidad, para fomentar y garantizar los derechos y desarrollo de los grupos originarios, y construir una ciudadanía que respecte, reconozca y se enriquezca de la interacción con la diversidad cultural, aquí también funcionan: la Dirección General de Ciudadanía Intercultural; la Dirección de Políticas para Pueblos Indígenas y el Grupo de Trabajo de Pueblos Indígenas (GTPI) (Ministerio de Cultura, 2014), (Olivera y Dietz, 2017).

Habíamos manifestado anteriormente que las políticas públicas deben abarcar todos los ámbitos: social, político, económico, cultural, ambiental, etc. La educación es una de esas dimensiones, y si es inclusiva mejor porque respondería a lo diverso; la educación inclusiva presta especial atención a estudiantes especiales en peligro de marginación o exclusión. Uno de los principales retos en Latinoamérica es ofrecer una educación de calidad; en el Perú, se ha empezado a simular con este objetivo, por un lado, en la educación básica, el Currículo Nacional contempla la inclusión de personas con necesidades educativas especiales y los lineamentos básicos sobre diversificación y adaptación curricular. Por otro lado, en la educación superior universitaria -Ley N³0220- exige una educación de calidad y para ello, las Universidades deben brindar un servicio de calidad. Así mismo, el Programa Nacional de Becas (PRONABEC), ha empezado a formular modalidades con el objetivo de beneficiar a más jóvenes indígenas desde 2012 de una manera muy lenta, quizá con la idea de buscar beneficio, es decir, formulación de políticas populistas de los gobiernos de turno. Desde el gobierno de Valentín Paniagua de noviembre del 2000 a julio del 2001, se han venido creando universidades 
interculturales como respuesta a la Propuesta Nacional para el Desarrollo de la Amazonía (2009). Actualmente, en el Perú hay cuatro universidades interculturales: la Universidad Nacional Intercultural de la Amazonía (UNIA), ubicada en el Departamento de Ucayali, Distrito de Yarinacocha, creada por ley 27250 del 10 de diciembre de 1999; la Universidad Nacional Intercultural Fabiola Salazar Leguía (UNIBAGUA), ubicada en el Departamento de Amazonas, Distritito de Bagua y creada por Ley N. ${ }^{\circ} 29614$, el 12 de noviembre de 2010; la Universidad Nacional Intercultural de Quillabamba (UNIQ), ubicada en el Departamento del Cusco, Distrito de Santa Ana y creada por Ley N. 29620 el 17 de noviembre de 2010; la Universidad Nacional Intercultural de la Selva Central Juan Santos Atahualpa (UNIJSA), ubicada en Departamento de Junín, Distrito de Chanchamayo y creada por Ley N. ${ }^{\circ} 29616$, el 17 de noviembre de 2010. La creación de estas universidades son respuestas populistas, porque ninguna de estas universidades muestra que tengan el "enfoque intercultural". Aún más, ninguna de estas universidades demuestra tener un modelo educativo, donde están plasmadas los elementos de su política educativa como universidad para solucionar los problemas de la comunidad, región y país. Por lo menos, la Universidad Intercultural de la Amazonia, no lo demuestra. Por otro lado, los planes curriculares de sus escuelas en la UNIA están bajo el enfoque por competencias, aplicados desde el 2011, y que ha sido replicado en el 2018, sin ningún análisis (evaluación integral) de si el currículo anterior ha dado buenos resultados o no, estos resultados pueden inferirse, pues la mayor parte de los egresados no puede sacar su título por deficiencias al momento de hacer una tesis, es decir, no demuestran competencia. Por otro lado, teniendo la experiencia, de la aplica- ción de este enfoque en la Educación Básica, aplicado desde 1995, seguimos demostrando deficiencias en compresión lectora y de ciencias como lo demuestran las pruebas PISA del 2015, el Perú ocupo el último lugar. Estos resultados quizá se deban a que, el enfoque por competencias es parametrizado como camisa de fuerzas, es decir, se sigue una receta sin tener en cuenta el desarrollo del pensamiento, razonamiento crítico y reflexivo que debe tener un profesional (Moreno y Soto, 2005).

Las deficiencias arriba mencionadas se deben también a otros factores, uno de ellos sino la más importante, es que los docentes no cumplen la función de instructor, porque el docente debe instruir, debe transmitir y enseñar a razonar sobre el conocimiento científico, sin subjetivismo e ideologías que crean el facilismo y tergiversan el sentido de la educación; además, el docente desconoce de nuevas metodologías pedagógicas, aquí hay que ser estrictos, respecto al verdadero sentido de la pedagogía, como es el arte de enseñar, no las pedagogías fomentadas por los pedagogos que creen que lo saben todo, sin embargo, no saben lo que enseñan. Por otro lado, el estado no se preocupa por especializar al docente universitario en general, becas a docentes universitarios, etc. Esto demuestra, que las políticas del estado peruano en el contexto intercultural son incipientes (Paya, 2010).

Las políticas de educación inclusiva deben ser transversales e ir acompañadas de otras políticas sociales, con una perspectiva multisectorial, para ello, se debe tener en cuenta cómo mejorar, por ejemplo: el nivel educativo, la alfabetización, formación docente, retención y prevención de la deserción escolar, población rural y pueblos originarios, becas, utilización y acceso a las TIC (Paya, 2010). 
Uno de los principales retos para el siglo XXI, frente a la diversidad cultural, creemos que es la educación, porque el conocimiento es poder, y eso lo sabe quiénes fomentan el capitalismo. Según la UNESCO la educación para el siglo XXI debe fundamentarse en: aprender a hacer, aprender a conocer, aprender a vivir juntos y aprender a ser. Diríamos con más énfasis en aprender hacer, es decir, todo individuo debe saber hacer bien lo aprendido en una universidad, si esto no sucede o demuestra, entonces el sistema universitario está fracasando y sobre todo quienes instruyen.

Hay que mirar la historia desde el punto de vista consecuente, para no cometer los mismos errores en el futuro, por ejemplo, uno de los errores gracias a los valores democráticos de entonces, fue la segunda guerra mundial, que costo millones de vidas. Y para no repetir esos errores es necesario el establecimiento de un sistema político que busque la libertad del hombre y la equidad en salud, educación y justicia (Ladrón y Navarro, 2009).

Hay diversos movimientos sociales que se han gestado en diferentes países de Latinoamérica con sus propias características, pero todos ellos tienen un común denominador las desigualdades radicales en lo social, económico, educativo, de salud, etc., y esto se desarrolla en supuestas democracias que aún no atienden las necesidades de gran parte de la sociedad. Democracias que han servido para el surgimiento de una clase dirigente millonaria y corrupta, que se mantiene por las constantes impregnación de ideologías, en los diversos campos y más aún en el sistema educativo, esto trae como consecuencia una pauperización del ser humano en todo su ser, es decir, se está generando zombis, que sirven para ciertos propósitos de quienes los formaron a través de sistemas educativos creados para esto. Nuestra generación no vera estas consecuencias, corresponderá, a las nuevas generaciones, resolver este problema que ojalá tenga solución. Necesitamos sistemas políticos superiores que le den libertad al ser humano dentro de la realidad. Téngase en cuenta que no estamos hablando de esta democracia en la que estamos inmersos, una democracia llena de ideologías y contradicciones (acentuado en de la posmodernidad) que necesariamente deben desaparecer (Bueno, 2004). Creemos que no todo está perdido, siempre después de atravesar un túnel hay luz. Los pueblos originarios han comenzado a participar en la gestación de políticas públicas en algunos estados de Latinoamérica, a través de la organización de partidos o agrupaciones políticas, por ejemplo: Movimiento $\mathrm{Pa}-$ chacuti, dirigido por Luis Macas, en Ecuador, y el Movimiento Político Winaq, en Guatemala, dirigido Rigoberta Menchú, o buscando alianzas entre organizaciones como es el caso del Movimiento al Socialismo (MAS), liderado por Evo Morales (Machaca, 2013).

Debemos agregar que el (MAS) hace su aparición en la escena política en 1995, convirtiendo en presidente de Bolivia a Evo Morales en el 2006. Desde entonces han paso 14 años 9 meses y el MAS ha ido generando un socialismo que se hace en la práctica, con resultados positivos para mejorar la calidad de vida de la Mayoría (poblaciones indígenas). Con un golpe de estado, Evo Morales es obligado a Renunciar 2019. Porque ocurrieron estos hechos, diríamos por un lado por el descuido de la formalidad (no respetar las normas y no haber creado un respaldo militar que apoye la revolución); y, por otro lado, no haber 
aprendido de la historia, en algunos países se iniciaron revoluciones socialistas, que hoy han desaparecido, y en otras de ellas sobrevive un hibrido entre socialismo y capitalismo. Porque el capitalismo internacional-nacional, no se va a quedar de brazos cruzados, siempre va estar minando para retomar al poder donde haya un intento de establecer gobiernos con prácticas socialistas. El objetivo que persigue el capitalismo Internacional-nacional, es la expropiación de los recursos (materia prima) de cada país, a costa de enriquecer a una clase dominante (minoría) por corrupción y mantener adormilada y embelesada a la clase dominada (mayoría) (Cabrera, et al., 2013)

\section{CONCLUSIONES}

La diversidad cultural, dentro del contexto de la modernidad actual, solo muestra las diferencias y desigualdades propias de un sistema capitalista monocultural y globalizante. Tal vez en el transcurso de este siglo XXI, las generaciones de jóvenes de esta diversidad de culturas puedan luchar por suprimir el sistema político actual y establecer un nuevo sistema político y social, y la interculturalidad desde el punto de vista objetiva tenga real y sostenida participación en esta construcción. Las políticas públicas interculturales, así como están pensadas perece ser excelentes iniciativas, que buscan el desarrollo de los pueblos originarios. Pero si estas siguen desarrollándose en el sistema capitalista actual, hay mucha duda que logren desarrollar los objetivos planificados. Porque el sistema capitalista va generando formasjurídicascapacesdetergiversarelverdadero valor de los elementos culturales de los pueblos originarios (por ejemplo, mercancías turísticas o patrimonio cultural de la humanidad).

Creemos que la interculturalidad funcional está contaminada o desvirtuada, porque fue desarrollada por y para el estado actual neoliberal. Debe tomarse una interculturalidad objetiva sin ideologías o el que haga sus veces, que permita descolonizar el poder del ser y del saber (conocimiento).

Una de las políticas públicas con mayor énfasis, debe ser en la esfera de la educación para el siglo XXI y debe fundamentarse en: aprender a hacer, aprender a conocer, aprender a vivir juntos y aprender a ser, con más realce en aprender hacer, es decir, todo individuo debe saber hacer lo aprendió en una universidad. Si esto no sucede entonces el sistema universitario está fallando y sobre todo quienes lo instruyen.

Creemos que una de las formas de ir fertilizando y preparado el terreno para la implantación de un nuevo sistema político y social, es generando partidos políticos. Y movimientos sociales capaces de incorporar a la interculturalidad como elemento estructural, asumir el poder y desarrollar liderazgo inclusivo.

\section{REFERENCIAS BIBLIOGRÁFICAS}

Aguiló, A. (2012). Interculturalidad, democracia y emancipación social: algunos retos para una teoría política intercultural. Revista Internacional de Filosofía. N.. 11. pp. 1-13. https://raco.cat/index.php/Astrolabio/article/view/238947. 
Aníbal, Q. (1a Ed.). (2014). Cuestiones y horizontes: de la dependencia histórico-estructural a la colinealidad/descolonialidad del poder. Ciudad Autónoma de Buenos Aires: CLACSO. ISBN 978-987-722-018-6.

Aguirre, J., Botero A. y Pavón, A. (2020). Neoliberalismo: análisis y discusión de su polisemia. Justicia, 25(37), pp. 109-124. https://doi.org/10.17081/just.25.37.3523.

Arroyo, M. (2013). La Educación Intercultural: un camino hacia la inclusión educativa. Revista de Educación Inclusiva, Vol. 6 (2). pp. 144-159.

Berrospi, M. (2015). Revalorando la interculturalidad del Varayoc en el desarrollo personal y social de los estudiantes de educación primaria de la Universidad Nacional Daniel Alcides Carrión sede Yanahuanca-Pasco. (Tesis doctoral). Universidad Nacional de Educación, Enrique Guzmán y Valle.

Bueno, G. (1a Ed.). (2004). Panfleto contra la democracia realmente existente. Madrid: la esfera de los libros.

Cabrera, F., Pop, A., Morales, Z., Chuji, M. y Mamani, C. (1a Ed.). (2013). Ciudadanía intercultural, aportes desde la participación politica de los pueblos indígenas en Latinoamérica. PNUID.https://www.undp.org.

Conde, M, (2017). Aplicación del ciclo 4MAT para facilitar la gestión de conocimiento en equipos de trabajo. (Tesis Doctoral). Universitat de les Illes Balears.

Cruz, M. (2010). Fundamentos normativos para las políticas públicas interculturales. Revista Perspectivas de Políticas Públicas. Año 3. N.o 5. pp. 121-140. ISSN 1853-9254

Daros, W. (2015). La creación de la modernidad nuevos deseos e intereses de la humanidad. Universidad del Centro Educativo Latinoamericano Rosario, Argentina. Invenio Vol.18 N. 34. pp.51-65. https://www.redalyc.org/articulo.oa?id=87739279005

Daza, H. (2010). La sociedad moderna. Revista Venezolana de Economía y Ciencias Sociales. N. ${ }^{\text {2 } 2 . ~ V o l . ~ 16 . ~ p p . ~ 61-83 . ~}$

Gonzales, O. (2007). Crítica de la interculturalidad: la construcción de un proceso en el marco de la modernización, Cuadernos Interculturales, Vol. 5, ․o. 9, pp. 63-89. https://www.redalyc.org/articulo.oa?id=55250905

González, M. y Sánchez, P. (2001). Minorías étnicas y movimientos separatistas en el mundo. México: Editorial Quimera. 
Hernández, M. (2007). Sobre los sentidos de "Multiculturalismo" e "Interculturalismo" Revista Ra Ximhai. Vol. 3. N.o 002.pp. 429-442. ISSN_1665.0441 México.

Ladrón, G. y Navarro, J. (2009). La educación intercultural como respuesta educativa del siglo XXI. Consejería de Educación y Cultura de la Región de Murcia, Didáctica Geográfica, 2. época 10, pp. 89-119. ISSN: 0210-492-X.

Lanegra I. (2016). Valor social e interculturalidad. Revista de Estudiantes de la Facultad de Gestión y Alta Dirección, PUCP, N.o 1, pp.39-44. https://revistas.pucp.edu.pe.

Laura, M. (2004). Reflexiones en torno a la interculturalidad. Cuadernos de Antropología Social. $\mathrm{N}^{\mathrm{o}} 19$, pp. 191-213.

Machaca, G. (2013). Hacia la inter culturalización de las políticas públicas, Fundación para la Educación en Contextos de Multilingüismo y Pluriculturalidad (FUNPROEIB Andes), ISEES N. ${ }^{\circ} 12$, pp. 17-30.

Ministerio de Cultura. (2014). Interculturalidad y políticas públicas Ministerio de Cultura, materiales de capacitación. Rescatado de www.cultura.gob.pe. 05/08/19.

Mendoza, V. (2012). Descolonización, Interculturalidad y Socialismo, El debate político en la Bolivia del siglo XXI. La Comuna Editores.

Millán, K. y Rincón, O. (2015). El asunto decolonial: conceptos y Perspectivas. Revista de historia, geografía, arte y cultura. Año 3. No. 5. Universidad Nacional Experimental Rafael María. Baralt Editorial/Editor.

Molina, J. (2012). Teoría democrática desde el paradigma de la interculturalidad. Tesis para optar al grado de doctor en filosofía iberoamericana. Universidad Centroamericana "José Simeón Cañas".

Moreno, P. y Soto, G. (2005). Una mirada reflexiva y crítica al enfoque por competencias. Educar. N. 35 Indd. pp.74-80. Rescatado de www.quadernsdigitals.net. 30/10/19.

Naciones Unidas. (2015). América Latina en agenda para el desarrollo después de 2015: reflexiones preliminares basadas en la trilogía de la igualdad. CEPAL. pp. 1-17.

Olivera, I. y Dietz, G. (2017). Educación superior y pueblos indígenas: marcos nacionales para contextualizar. Antropológica/año XXXV, Vol.35 No.39, pp. 7-39. http://dx.doi.org/10.18800/anthropologica.201702.001. 
Paya, A. (2010). Políticas de educación inclusiva en América Latina Propuestas, realidades y retos de futuro. Revista Educación Inclusiva. Vol. 3, N. ํ 2. pp. 1-16. ISSN-1889-4208.

Pérez, R. (2016). Procesos interculturales en una comunidad indígena en Chiapas desde una mirada EMIC: realidades y desafíos Sinéctica. Revista Electrónica de Educación, N.o. 47, pp. 1-16. ISSN: 1665-109X

Reguillo, R. (2005). Horizontes fragmentados, comunicación, cultura, pospolítica. El (des) orden global y sus figuras. México: Instituto Tecnológico de Estudios Superiores de Occidente.

Turner, M. y Hulme, D. (1997). Governance, Administration and Development: Making the State Work. Macmillan Publishers Limited. https://oi.org/10.1007/978-1-349-25675-4

Vidal, P. (1a Ed.). (2019). Neoliberalismo, Neodesarrollismo y Socialismo bolivariano. Modelos de desarrollo y Políticas públicas en América Latina. Santiago de Chile. 